\title{
Estimating Dynamic Lung Images from High-Dimension Chest Surface Motion Using 4D Statistical Model
}

\author{
Tiancheng He, Zhong Xue*, Nam Yu, Paige L. Nitsch, Bin S. Teh, \\ and Stephen T. Wong \\ Houston Methodist Research Institute, Weill Cornell Medical College, Houston, TX, US \\ zxue@houstonmethodist.org
}

\begin{abstract}
Computed Tomography (CT) has been widely used in image-guided procedures such as intervention and radiotherapy of lung cancer. However, due to poor reproducibility of breath holding or respiratory cycles, discrepancies between static images and patient's current lung shape and tumor location could potentially reduce the accuracy for image guidance. Current methods are either using multiple intra-procedural scans or monitoring respiratory motion with tracking sensors. Although intra-procedural scanning provides more accurate information, it increases the radiation dose and still only provides snapshots of patient's chest. Tracking-based breath monitoring techniques can effectively detect respiratory phases but have not yet provided accurate tumor shape and location due to low dimensional signals. Therefore, estimating the lung motion and generating dynamic CT images from real-time captured high-dimensional sensor signals acts as a key component for image-guided procedures. This paper applies a principal component analysis (PCA)-based statistical model to establish the relationship between lung motion and chest surface motion from training samples, on a template space, and then uses this model to estimate dynamic images for a new patient from the chest surface motion. Qualitative and quantitative results showed that the proposed high-dimensional estimation algorithm yielded more accurate 4D-CT compared to fiducial marker-based estimation.
\end{abstract}

Keywords: high-dimensional respiratory motion estimation, statistical model.

\section{Introduction}

Medical imaging has been widely used not only for diagnosis but also for intervention, radiotherapy, and surgery guidance. The key for image-guided procedures is to visualize patient's anatomy and operative devices simultaneously in real-time. However, in many cases real-time imaging is available in coherent with device tracking. In percutaneous lung intervention, electromagnetic or optical tracking can be used for detecting the interventional probes, but there is lack of real-time imaging technique for navigation. Using static pre-procedural CT is not able to solve the discrepancies between the CT and patient's respiratory stages. Thus, monitoring respiratory phases

\footnotetext{
* Corresponding author. 
has been the major focus in dealing with breathing or poor reproducibility of breathholding. Motion compensation also becomes a major challenge for more accurate guidance: it is highly desirable that a precise lung motion model can be used as the roadmap for guiding the intervention during each breath holding cycle.

Recent works for characterizing lung motion can be classified to three categories. The first is lung motion deformation modeling with registration [1-3], but they only construct the respiratory patterns and do not achieve motion modeling/estimation. The second group uses individual patient's dataset [4], e.g., first extracting lung motion in pre-procedural scans and then applying this model during procedures (radiotherapy or intervention). Finally, statistical model can be applied to incorporate both group and individual information for motion modeling. In this way, respiratory patterns trained from a large number of subjects can be used to estimate the dynamic images of individuals, even when the 4D-CT images are not available for them $[5,6]$. For example, one can use the motion of a patient's external features in combination with a motion model to compensate for internal respiratory motion [7-10]. One challenge is that the limited dimension of respiratory sensor signals may not reflect the high-dimensional lung motion accurately. Although monitoring respiratory phases well, they may not estimate accurate lung tumor location and shape dynamics. With new computer vision technology, the non-contact vision-based motion monitoring devices can be used for detecting the high-dimensional chest surface motion [11], and it would be promising to estimate dynamic lung motion by statistical model-based prediction between highdimensional chest surface and lung motion vectors.

In this paper, we propose to estimate dynamic lung images using a 4D statistical model. In the training stage, after performing longitudinal registration of the 4D-CT data of each training subject, the respiratory motion fields are obtained. Then, all the images, lung motion fields, and chest surface motion for all the subjects are aligned onto a template space for training a statistical model. The statistical model is constructed using the high-dimensional 4D motion data to establish the relationship between the chest surface motion and lung respiratory motion in the template space. During the motion estimation stage, the chest surface motion and 3D-CT of a new patient will be captured and transformed onto the template space, and we apply the statistical model to predict the patient-specific lung field motion from the chest surface signals. Finally, the estimated dynamic lung images are transformed to the patient image space for guidance.

In experiments, we used 4D-CT data from thirty lung cancer subjects undergoing radiation therapy planning to construct the statistical model and applied the model to additional 10 subjects for validation. We segmented the tumors and calculated the DICE coefficients between the real testing images and the estimated images. In addition, we also compared the accuracy for estimating deformations with the previous fiducial-based estimation. The results showed that higher accuracy can be achieved with high-dimensional estimation.

\section{$2 \quad$ Method}

The proposed algorithm consists of training and prediction stages. In the training stage, the respiratory motion fields and surface motion vectors for each training 
subject are aligned onto the template space, and the PCA model is applied to estimate the joint distribution of the 4D high-dimensional data. In the motion prediction stage, the 3D-CT and chest surface motion of a new subject are obtained, and by registering these data onto the template space, the motion estimation is performed to generate a series of lung deformations based on the chest surface motion vectors using the statistical model as the constraints. The serial deformation fields are then transformed onto the patient's 3D-CT image space to generate the serial CT images for the patient.

\subsection{Image Registration and Motion Vector Transformation}

For intra-subject registration the main consideration is temporal consistency of motion vectors extracted from the training 4D-CT data. Hence, the joint 4D segmentation and registration algorithm [12] is used. After registration, the longitudinal deformations $\mathbf{f}_{1 \rightarrow 2}^{S}, \mathbf{f}_{1 \rightarrow 3}^{S}, \ldots, \mathbf{f}_{1 \rightarrow M}^{S}$, for serial images, $I_{1}^{S}, I_{2}^{S}, \ldots, I_{M}^{S}$, of subject $s$ with $M$ respiratory phases are obtained. For inter-subject registration, the baseline (exhale) images between the subject and template, $I_{1}^{S}$ and $I_{1}^{T}$ are registered [13], resulting a global affine transformation $G_{T \rightarrow S}$ and a deformation field $\mathbf{f}_{T \rightarrow S}$. So, the subject images are first transformed using $G_{T \rightarrow S}^{-1}$ and using field $\mathbf{f}_{T \rightarrow S}$ onto the template space.

To transform the respiratory motion fields onto the template space, the motion vectors for each subject need to be scaled and oriented. Thus, the following global and local re-orientation processes between the subject and the template are applied to $\mathbf{f}_{1 \rightarrow 2}^{S}, \mathbf{f}_{1 \rightarrow 3}^{S}, \ldots, \mathbf{f}_{1 \rightarrow M}^{S}$, resulting the respiratory motion of the same subject on the template space. Denoting $g_{T \rightarrow S}$ the re-orientation matrix of $G_{T \rightarrow S}$ without translation, the respiratory motion of subject $s$ is calculated as $\mathbf{f}_{1 \rightarrow m}^{s \prime}=g_{T \rightarrow S}^{-1} \mathbf{f}_{1 \rightarrow m}^{S}, m=2, \ldots M$. Then, a local re-orientation is achieved by,

$$
\mathbf{u}_{1 \rightarrow m}^{S}(\mathbf{x})=R_{T \rightarrow S}^{-1}(\mathbf{x}) \mathbf{f}_{1 \rightarrow m}^{S \prime}\left(\mathbf{x}+\mathbf{f}_{T \rightarrow S}(\mathbf{x})\right),
$$

where $R_{T-S}(\mathbf{x})$ is the local rotation matrix at template voxel $\mathbf{x}$, calculated from the Jacobian matrix $J_{T \rightarrow S}(\mathbf{x})$ of $\mathbf{f}_{T \rightarrow S}(\mathbf{x})$ as $D_{T \rightarrow S}(\mathbf{x})=I+J_{T \rightarrow S}(\mathbf{x})$,

$$
R_{T \rightarrow S}(\mathbf{x})=\left(D_{T \rightarrow S}(\mathbf{x}) D_{T \rightarrow S}^{\mathrm{T}}(\mathbf{x})\right)^{-1 / 2} D_{T \rightarrow S}(\mathbf{x}) .
$$

In this way, all the longitudinal deformation fields calculated from the 4D-CT training samples are transformed onto the template space. Similar operations are applied to the chest surface motion vectors.

For a new subject $P$, the global affine transformation $G_{T \rightarrow P}$ (reverse as $G_{P \rightarrow T}$ ) and deformation field $\mathbf{f}_{T \rightarrow P}$ (reverse as $\mathbf{f}_{P \rightarrow T}(\mathbf{x})$ ) can be applied between the template and patient spaces. So the estimated the respiratory motion for a patient, $\mathbf{u}_{1 \rightarrow m}^{P}$, in the template space, can be transformed back to the patient space using,

$$
\mathbf{f}_{1 \rightarrow m}^{P}(\mathbf{x})=g_{T \rightarrow P} \circ R_{T-P}(\mathbf{x}) \mathbf{u}_{1 \rightarrow m}^{P}\left(G_{P \rightarrow T}\left(\mathbf{x}+\mathbf{f}_{P \rightarrow T}(\mathbf{x})\right)\right) .
$$

\subsection{Statistical Model-Based Motion Estimation}

After obtaining the respiratory motion field $\mathbf{u}_{1 \rightarrow m}^{s}, s=1, \ldots, N ; m=2, \ldots, M$, and the chest surface motion $\mathbf{v}_{m}^{s}$, for subject $s$ with $M$ respiratory phases, we construct the 
statistical model of the longitudinal fields and motion vectors in the template space using PCA by rearranging them into column vectors. For simplicity, we still use the same symbols to represent them. Under this convention, the serial lung motion fields and chest surface motion vectors are represented as $\mathbf{u}^{s}$ and $\mathbf{v}^{s}$, respectively, and their mean vectors over all the $\mathrm{N}$ training samples are denoted as $\overline{\mathbf{u}}$ and $\overline{\mathbf{v}}$. According to the PCA model, a new vector can be calculated by,

$$
\left[\begin{array}{l}
\mathbf{u} \\
\mathbf{v}
\end{array}\right]=\left[\begin{array}{l}
\overline{\mathbf{u}} \\
\overline{\mathbf{v}}
\end{array}\right]+\mathrm{M}\left[\begin{array}{l}
\mathbf{b}_{u} \\
\mathbf{b}_{v}
\end{array}\right] .
$$

$\mathrm{M}$ is the matrix formed by the eigenvectors of the covariance matrix corresponding to $K$ largest eigenvalues. Thus, given a new feature vector $\mathbf{b}=\left[\mathbf{b}_{u}^{T}, \mathbf{b}_{v}^{T}\right]^{T}$, the lung motion field and the surface motion vector can be generated using Eq. (4). The multidimensional Gaussian distribution is used to model these feature vectors,

$$
p(\mathbf{b})=\frac{1}{\sigma} \mathrm{e}^{\left\{-\sum_{k=1}^{K} b_{k}^{2} / 2 \lambda_{k}\right\}} .
$$

To estimate the lung respiratory motion field $\mathbf{u}^{P}$ from the chest surface motion $\mathbf{v}^{P}$, we need to solve the best the feature vector $\mathbf{b}$, so that the estimated $\mathbf{v}$ matches the patient's chest surface motion $\mathbf{v}^{P}$, and at the same time, the prior distribution is maximized [14]. The energy function is defined as,

$$
E(\mathbf{b})=\left\|\mathbf{v}^{P}-\overline{\mathbf{v}}-{ }_{v} \mathbf{b}\right\|^{2}+\xi \sum_{k=1}^{K} b_{k}^{2} / 2 \lambda_{k},
$$

where $_{v}$ is the matrix formed by the last $H$ rows of $\mathbf{M}$, with $H$ the dimension of $\overline{\mathbf{v}}$. $\xi$ is the weighting factor for constraining $\mathbf{b}$ with the prior distribution. After $\mathbf{b}$ is estimated, the lung motion vector $\mathbf{u}^{P}$ can be calculated by $\mathbf{u}^{P}=\overline{\mathbf{u}}+{ }_{u} \mathbf{b}_{P}$, with ${ }_{u}$ as the top part of $\mathrm{M}$ corresponding to the lung respiratory motion field.

\subsection{Estimating Patient-Specific Lung Motion Fields}

Vision-based monitoring devices [11] are used to record patient's chest surface motion. The coordinates of chest surface from the monitoring devices are transferred to the scanned CT image space after camera calibration and surface-based registration. Suppose the serial surfaces are represented by $\mathbf{d}_{t}$, with $t$ as time. According to the exhale and inhale status of the surface signals, we temporally divide surface motion vectors in a breathing cycle to $M$ phases, denoted as $\mathbf{w}_{m}=\mathbf{d}_{m}-\mathbf{d}_{1}, m=2, \ldots, M$. By substituting $\mathbf{w}_{m}$ to $\mathbf{f}_{1 \rightarrow m}^{s}$ in Eq. (1), the chest surface motion vector on the template space can be calculated, which gives $\mathbf{v}^{P}$ as in Eq. (6). After optimizing Eq. (6) for the best feature vector $\mathbf{b}$, the lung respiratory fields $\mathbf{u}^{P}$ are estimated, which shows how the lung deforms during a respiratory cycle for the patient in the template space. Finally, we apply Eq. (3) to transform the deformation fields $\mathbf{u}^{P}$ from the template space to the patient space, and a series of CT images can be generated by deforming the 3D CT image of the patient. Intermediate images can also be obtained by sampling the estimated longitudinal deformations if more than $\mathrm{M}$ images are needed.

Ideally, the above estimation should be performed in real-time. However, because of the computational load for transformations between the template and the patient 
spaces, as well as the statistical model-based lung deformation field estimation, it took about 3 minutes for the estimation. Therefore, for real clinical use, we record the chest surface signals for multiple cycles, and pre-calculate CT serial images based on the recorded signals. During the guidance, a least squares error method can be used to select the best CT image that matches the current chest surface signals.

\section{Results}

In experiments, 4D-CT datasets from forty lung cancer patients undergoing radiotherapy planning were used to evaluate the performance of the estimation accuracy. $\mathrm{Pa}$ tients were with smaller lesions to eliminate the registration and training errors for motion modeling. The 4D-CT images for each subject consist of ten respiratory phases with the first and the last images to be the exhale images and the fifth and the sixth are the inhale images. The in-plane resolution of each image is $0.98 \mathrm{~mm} \times 0.98 \mathrm{~mm}$ and the slice thickness is $1.5 \mathrm{~mm}$. One subject was selected as the template, 29 were used for training and 10 were used for testing. A template generated by group-wide registration may be used in the future to minimize the bias toward template images. For the testing sample, the chest surface signals derived from 4D-CT and the exhale CT were used for estimating CTs of other phases, and the estimation results were compared with the known 4D-CT. Our algorithm was implemented on a DELL workstation with 4 Inter 2.5GHz dual core Quad Q9300 processors and 8GB RAM. The time for training was around 1 hour, and that for testing each case was less than 3 minutes.

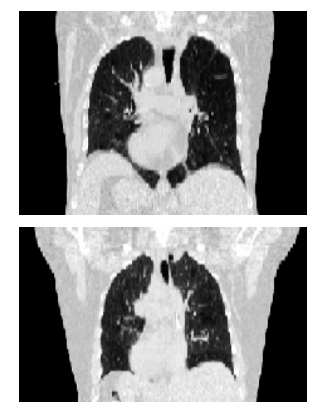

(a)
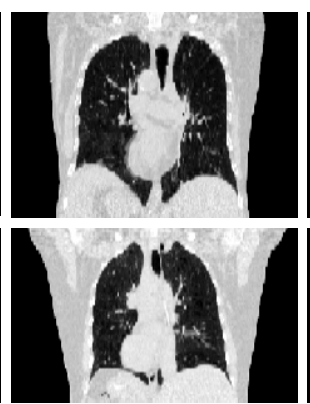

(b)

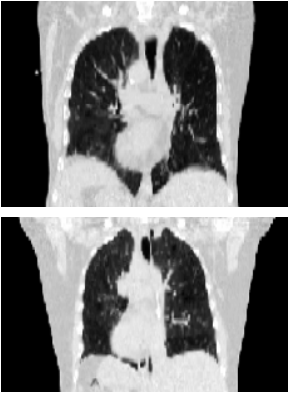

(c)
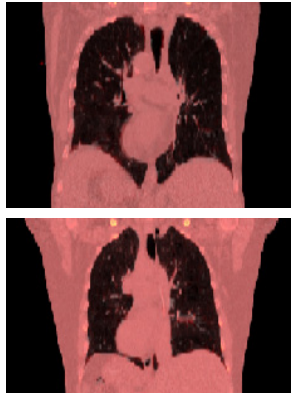

(d)

Fig. 1. Examples of the estimation results. The exhale (a) and inhale (b) images of two subjects; (c) estimated inhale images; (d): Overlap of predicted and real inhale images.

Fig. 1 shows two examples of the results. The real exhale and inhale images of the subjects are shown in column (a) and (b), and column (c) gives the estimated inhale images by using the inhale chest surface signals. The images in column (d) show the overlapping between the estimated inhale images and the real inhale images. It can be seen that the estimated inhale images match the real inhale image well.

To quantitatively evaluate the performance of the algorithm, we compared the proposed surface-based estimation with the estimation based on EM-tracked chest fiducials [9]. The difference is that for chest fiducial points, four fiducial signals were 
used to track the chest motion, while for the propose method, the entire chest surface was applied for lung respiratory motion estimation. The idea is that high-dimensional deformation is involved in the prediction problem, and compared to fiducial signals with four sensors, high-dimensional chest surface motion would provide more accurate estimation. For the fiducial-based estimation, because of the limited number of fiducials, it may be feasible to predict the respiratory phases and to monitor the respiratory motion during intervention, but it may not have sufficient degree-of-freedom to predict the precise shape and location of the internal anatomical structures, such as lung field, bone, diaphragm, and tumor, etc.

With 30 datasets for training and 10 for testing, we can compute the quantitative performance as how well the method recovers the longitudinal deformations for each testing image series. For each testing subject, we register the 4D-CT images and obtain the longitudinal deformations $\mathbf{f}_{1 \rightarrow m}^{*}(\mathbf{x})$. After estimation, the estimated longitudinal deformations are denoted as $\hat{\mathbf{f}}_{1 \rightarrow m}(\mathbf{x}), m=2, \ldots, M$. The average lung respiratory motion estimation error for each subject can be calculated as,

$$
\varepsilon=\frac{1}{|\Omega|} \sum_{m=2, \ldots M} \sum_{\mathbf{x} \in \Omega}\left|\mathbf{f}_{1 \rightarrow m}(\mathbf{x})-\hat{\mathbf{f}}_{1 \rightarrow m}(\mathbf{x})\right|,
$$

where $\Omega$ represents the domain of the voxels of the first time-point image of each subject. $|\Omega|$ is the number of voxels.

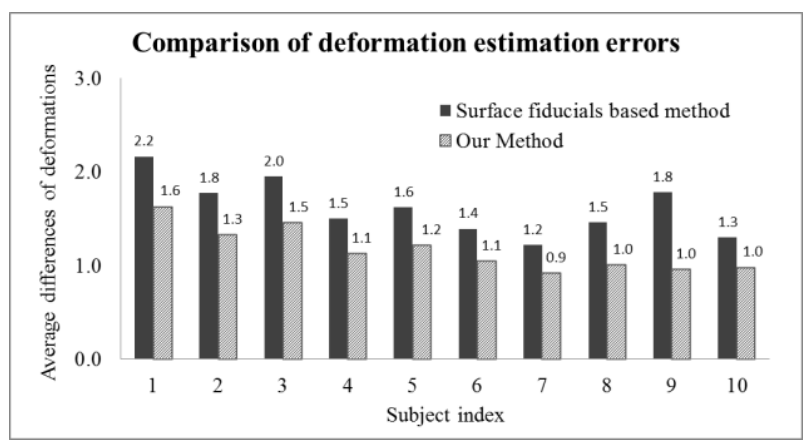

Fig. 2. Average errors for lung motion estimation

Fig. 2 plots the average estimation errors for each subject. The solid bars are the errors for fiducial-based estimation, and the shaded bars show the estimation errors using chest surface-based estimation. The estimation errors using our proposed surface-based estimation method are between $0.92 \mathrm{~mm}$ and $1.63 \mathrm{~mm}$ with an average of $1.17 \mathrm{~mm}$, while the errors using fiducial-based estimation method are between 1.22 $\mathrm{mm}$ and $2.16 \mathrm{~mm}$ with an average of $1.61 \mathrm{~mm}$. Overall, the results show that surfacebased estimation outperforms the fiducial-based estimation. Thus, for current clinically used techniques such as respiratory belts (one-dimensional signal) and fiducial-based breath tracking (limited number of signals), although they can be used for monitoring the respiratory phases, they may be limited for predicting the lung motion. On the other hand, using the chest surface-based statistical motion estimation, we could obtain more accurate estimation. 
In another evaluation, we calculated how well the shape of lung tumor is estimated. Fig. 3 shows some sample results. Fig. 3 (a) is the inhale CT for a subject, and Fig. 3 (b) is the exhale CT. Their overlapping is shown in Fig. 3 (c). To measure the tumor estimation accuracy, we estimate the inhale images form exhale images and compare quantitatively whether the estimated tumor shape in the estimated inhale phase matches that in the original inhale image. Fig. 3 (d-f) shows the images of three other subjects with red curves as the manually marked tumor from the original inhale images, and the green curves show the estimated tumor shapes. We can see that the tumor positions and shapes under different respiratory phases can be estimated well. The DICE coefficient was used for quantitatively measuring estimation results of tumor positions. In our experiments, the average value of DICE coefficients over the 10 testing subjects are $88.1 \% \pm 2.8 \%$ using the proposed algorithm, while the results are $82.6 \% \pm 5.7 \%$ for fiducial-based estimation. Overall, these results demonstrate promising dynamic lung motion modeling.
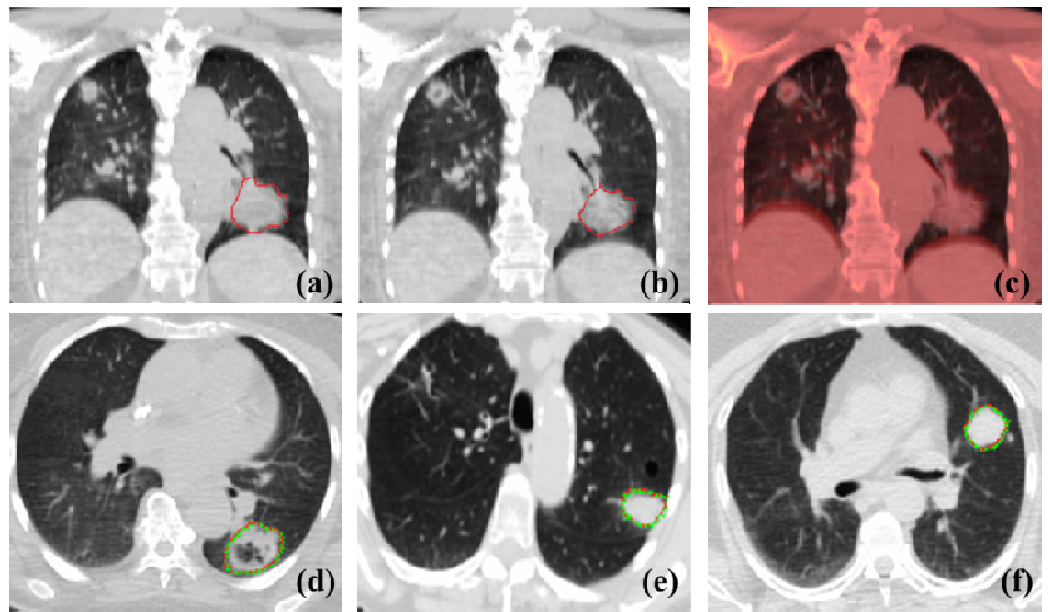

Fig. 3. Lung tumor estimation with dynamic modeling. (a) Exhale CT; (b) inhale CT; (c) overlaying (a) and (b); (d-f) comparisons of tumor estimation between actual and estimated CT images for three additional subjects. Red: real tumor outline; green: estimated tumor.

\section{Conclusion}

A statistical model-based patient-specific 4D-CT estimation method is proposed for respiratory motion compensation. After registering the training data including 4D-CT and chest surface motion vectors for each subject onto the template space, a statistical model is trained, and the lung respiratory motion for new subject can be estimated from the chest surface motion vector. Finally, the estimated serial deformations are transformed onto the patient space. Quantitative validation using lung deformation field estimation errors and DICE coefficients for tumor shape estimation accuracy showed that the proposed surface-based estimation outperforms the fiducial-based 
prediction. The proposed dynamic image estimation method could be used to guide the delivery of radiation beams to the moving lung tumor or to help navigate the interventional device during percutaneous lung cancer intervention, e.g., image-guided biopsy and radiofrequency ablation.

\section{References}

1. Sundaram, T.A., Avants, B.B., Gee, J.C.: A dynamic model of average lung deformation using capacity-based reparameterization and shape averaging of lung MR images. In: Barillot, C., Haynor, D.R., Hellier, P. (eds.) MICCAI 2004. LNCS, vol. 3217, pp. 10001007. Springer, Heidelberg (2004)

2. Vandemeulebroucke, J., Rit, S., Kybic, J., Clarysse, P., Sarrut, D.: Spatiotemporal motion estimation for respiratory-correlated imaging of the lungs. Med. Phys. 38, 166-178 (2011)

3. Handels, H., Werner, R., Schmidt, R., Frenzel, T., Lu, W., Low, D., Ehrhardt, J.: 4D medical image computing and visualization of lung tumor mobility in spatio-temporal CT image data. Int. J. Med. Inform. 76(suppl. 3), S433-S439 (2007)

4. Wu, G., Wang, Q., Lian, J., Shen, D.: Estimating the 4D respiratory lung motion by spatiotemporal registration and building super-resolution image. In: Fichtinger, G., Martel, A., Peters, T. (eds.) MICCAI 2011, Part I. LNCS, vol. 6891, pp. 532-539. Springer, Heidelberg (2011)

5. Klinder, T., Lorenz, C., Ostermann, J.: Prediction framework for statistical respiratory motion modeling. In: Jiang, T., Navab, N., Pluim, J.P.W., Viergever, M.A. (eds.) MICCAI 2010, Part III. LNCS, vol. 6363, pp. 327-334. Springer, Heidelberg (2010)

6. Ehrhardt, J., Werner, R., Schmidt-Richberg, A., Handels, H.: Statistical modeling of 4D respiratory lung motion using diffeomorphic image registration. IEEE Trans. Med. Imag. 30, 251-265 (2011)

7. Lu, W., Song, J.H., Christensen, G.E., Parikh, P.J., Zhao, T., Hubenschmidt, J.P., Bradley, J.D., Low, D.A.: Evaluating lung motion variations in repeated 4D CT studies using inverse consistent image registration. Int. J. Radiat. Oncol. 66, S606-S607 (2006)

8. Santelli, C., Nezafat, R., Goddu, B., Manning, W.J., Smink, J., Kozerke, S., Peters, D.C.: Respiratory Bellows Revisited for Motion Compensation: Preliminary Experience for Cardiovascular MR. Magnet. Reson. Med. 65, 1098-1103 (2011)

9. He, T., Xue, Z., Xie, W., Wong, S.T.C.: Online 4-D CT estimation for patient-specific respiratory motion based on real-time breathing signals. In: Jiang, T., Navab, N., Pluim, J.P.W., Viergever, M.A. (eds.) MICCAI 2010, Part III. LNCS, vol. 6363, pp. 392-399. Springer, Heidelberg (2010)

10. He, T., Xue, Z., Lu, K., Valdivia y Alvarado, M., Wong, K.K., Xie, W., Wong, S.T.: A minimally invasive multimodality image-guided (MIMIG) system for peripheral lung cancer intervention and diagnosis. Comput. Med. Img. Grap. 36, 345-355 (2012)

11. Tan, K.S., Saatchi, R., Elphick, H., Burke, D.: Real-time vision based respiration monitoring system. In: 7th International Symposium on Communication Systems Networks and Digital Signal Processing, pp. 770-774 (2010)

12. Xue, Z., Wong, K., Wong, S.T.C.: Joint registration and segmentation of serial lung CT images for image-guided lung cancer diagnosis and therapy. Comput. Med. Img. Grap. 34, 55-60 (2010)

13. Alexander, D.C., Pierpaoli, C., Basser, P.J., Gee, J.C.: Spatial transformations of diffusion tensor magnetic resonance images. IEEE Trans. Med. Imag. 20, 1131-1139 (2001)

14. Davatzikos, C., Shen, D., Mohamed, A., Kyriacou, S.K.: A framework for predictive modeling of anatomical deformations. IEEE Trans. Med. Imag. 20, 836-843 (2001) 